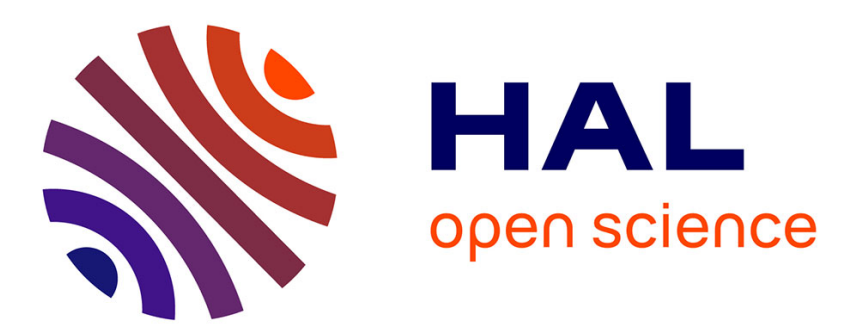

\title{
Performance of a thermally deformable mirror for correction of low-order aberrations in laser beams
}

M. Kasprzack, B. Canuel, F. Cavalier, R. Day, E. Genin, J. Marque, D. Sentenac, G. Vajente

\section{- To cite this version:}

M. Kasprzack, B. Canuel, F. Cavalier, R. Day, E. Genin, et al.. Performance of a thermally deformable mirror for correction of low-order aberrations in laser beams. Applied optics, 2013, 52, pp.2909-2916. 10.1364/AO.52.002909 . in2p3-00815277

\section{HAL Id: in2p3-00815277 \\ https://hal.in2p3.fr/in2p3-00815277}

Submitted on 18 Apr 2013

HAL is a multi-disciplinary open access archive for the deposit and dissemination of scientific research documents, whether they are published or not. The documents may come from teaching and research institutions in France or abroad, or from public or private research centers.
L'archive ouverte pluridisciplinaire HAL, est destinée au dépôt et à la diffusion de documents scientifiques de niveau recherche, publiés ou non, émanant des établissements d'enseignement et de recherche français ou étrangers, des laboratoires publics ou privés. 


\title{
Performance of a thermally deformable mirror for correction of low order aberrations in laser beams
}

\author{
Marie Kasprzack ${ }^{1,2, *}$, Benjamin Canuel ${ }^{2}$, Fabien Cavalier ${ }^{1}$, Richard Day ${ }^{2}$, \\ Eric Genin ${ }^{2}$, Julien Marque ${ }^{2}$, Daniel Sentenac ${ }^{2}$, Gabriele Vajente ${ }^{3}$ \\ 1 Laboratoire de l'Accelerateur Lineaire, \\ Centre Scientifique d'Orsay, Building 200, 91898 Orsay, France \\ 2 European Gravitational Observatory, \\ Via E. Amaldi, 56021 S. Stefano a Macerata, Cascina (PI), Italy \\ 3 Sezione di Pisa, Istituto Nazionale di Fisica Nucleare, \\ Via F. Buonarroti 2, 56127 Pisa, Italy \\ *Corresponding author: kasprzac@lal.in2p3.fr
}

\begin{abstract}
The thermally deformable mirror is a device aiming at correcting beamwavefront distortions for applications where classical mechanical methods are precluded by noise considerations, like in advanced gravitational wave interferometric detectors. This moderately low-cost technology can be easily implemented and controlled thanks to the good reproducibility of the actuation. By using a flexible printed circuit board technology, we demonstrate experimentally that a device of 61 actuators in thermal contact with the back surface of a HR mirror is able to correct the low-order aberrations of a laser beam at $1064 \mathrm{~nm}$ and could be used to optimize the mode matching into Fabry-Perot cavities. (c) 2013 Optical Society of America
\end{abstract}

OCIS codes: $110.1080,140.3300,230.4040,140.6810,350.6830$

\section{Introduction}

Interferometer gravitational wave detectors are known to be extremely sensitive to thermal lensing and elastic deformations of their core optics, even if the absorption of the coatings is at the level of $1 \mathrm{ppm}$. These issues have been extensively studied before the construction of GEO600, Virgo and LIGO [1-3]. The most recent theoretical overview is available in [4]. These issues have given rise to a new family of adaptive optics correction systems. Indeed, 
the particularly stringent requirements impose to avoid any mechanical contact with the optics, and in consequence ruled out the use of piezoelectric actuators. The first conceptual proposals and proof of principle experiments were made between 2000 and 2004 [5-8]. They targeted the uniformity of the temperature distribution in the optics by different means. Ring heaters $[9,10]$, external pump beams [11,12] and radiative heaters [13] were the first corrective systems tested and inplemented in the interferometer gravitational wave detectors.

Actually, not only the core optics of the Fabry-Perot cavities of gravitational wave detectors require adaptive optics corrections. Indeed, the beam coupling into these cavities can also be optimized using adaptive optics at the input of the interferometer. In advanced gravitational wave detectors, the coupling efficiency is required to be higher than $99 \%$ at the input of the interferometer [14]. Moreover, when the interferometer employs squeezed light injection [15], its efficiency depends strongly on the mismatching losses between the various cavities that are on the squeezed light path [16].

The experience gathered with core optics thermal effects gave birth then to a second family of adaptive optics systems aimed at optimizing the beam coupling into Fabry-Perot cavities. First a novel method was proposed for mismatching sensing [17] together with a passive method for correction [18]. The first real adaptive optics system was proposed in [19,20]. Another system using a segmented heater [21] was presented later on for its implementation in advanced LIGO.

Recently, a new concept of Thermally Deformable Mirror (TDM) with proof of principle was proposed in [22]. This device is designed to be used at the input of the interferometer. It is a complementary device to the injection mode matching optics. It can further improve the matching of the fundamental mode of the laser beam into the interferometer. This technology is more efficient than [21] since the correction is directly applied in the laser beam path. We propose here to measure the optical performance of such a solution.

We first describe the prototype and the setup in section 2, characterize the actuator response in section 3. Then we describe in section 4 the characteristics of the implemented control loop, and finally present the performances in term of amplitude of correction and accuracy limitations in section 5. In section 5.B we finally discuss how the mode matching into a Fabry-Perot cavity could be improved by using such a device.

\section{Experimental work conditions}

\section{A. Principle of actuation of a thermally deformable mirror}

The properties of an optical substrate change locally with temperature. This effect, generally a source of distortions in the laser beam propagation, can be exploited to correct the beam aberrations. The optical path length experienced by a laser beam passing through a substrate 
can be thermally controlled as explained in [22] and as shown in figure 1. Based on this principle, the TDM is a mirror in thermal contact with a body whose temperature can be tuned. It can be a resistor as proposed in [22] which is locally dissipating heat in the substrate. The index of refraction is then modified by the temperature increase. The active area is composed of an array of resistors. The resistors are put in contact with the high reflective face (HR) of a mirror whereas the anti-reflective coating (AR) allows the beam to propagate through the substrate reducing as much as possible the losses associated with the first surface. Such a device allows to control the wavefront of a laser beam.

\section{B. Prototype description}

All the tests presented here are realized with a prototype of the TDM designed to correct the aberrations of a $2.6 \mathrm{~mm}$ radius beam. The actuators, made of resistive layers of $18 \mu \mathrm{m}$ copper in contact with insulating layers of polyimide (Dupont Pyralux AP), are printed onto a multilayer flex circuit. The materials are designed to be used at high temperature and could be manufactured to guarantee no outgassing of the circuit board under vacuum. Such a technology allows to have a high density of resistors: the actuation pattern is composed of 61 actuators of $0.9 \times 0.9 \mathrm{~mm}^{2}$ each (see figure 2). The resistors have a mean value of $400 \mathrm{Ohms}$ with a standard deviation of 100 Ohms for the whole sample. The array of resistors can be put in contact with any kind of mirror substrate. Here we use a commercial fused silica, 2 inch diameter and $9.53 \mathrm{~mm}$ thick mirror with dielectric coatings. The first surface is coated with an AR coating and the array of resistors is acting on the surface with a HR coating for $1064 \mathrm{~nm}$. An interaction layer, like a glue or thermal paste, provides a good contact between the resistors and the HR surface of the substrate.

\section{C. Setup description}

The setup shown in figure 3 has been used to characterize the prototype. The tests are made in air. The TDM reflects the laser beam from a Nd:YAG NPRO laser $(\lambda=1064 \mathrm{~nm})$. A beam expander that generates a $2.6 \mathrm{~mm}$ radius beam ensures a complete coverage of the TDM active surface. The phase modifications are imaged using a two-lens telescope and measured with a wavefront sensor from Phasics, based on the lateral shearing interferometry principle [23]. This instrument does not require a reference beam for relative phase measurements. It has a maximum precision of $3 \mathrm{~nm}$ RMS. The tests were made with a $7 \mathrm{~mm}$ diameter pupil: the working aperture is inside the mirror actuation space (see figure 2) to benefit from the actuation on the pupil periphery as recommended in $[27,28]$.

The acquisition rate has been chosen to be 1 frame per second as the response time of the system is of a few seconds (see section 3). A computer processes the data with a MATLAB program to determine the required current to be applied on the resistors according to the 
algorithm presented in section 4. Then, a set of 61 DACs (Digital Analog Converters) controls the currents sent to the array of resistors. Each resistor is individually delivered to a specific current value. For the characterization tests, the DACs have a working range limited to 8 $\mathrm{mA}$.

When the whole dynamic is used, the maximum focus that can be corrected by the system is evaluated at around +/- $30 \mathrm{~m}$ ( $44 \mathrm{~nm}$ RMS) on a $7 \mathrm{~mm}$ pupil and the tilts can be corrected by up to $50 \mu \mathrm{rad}$. In the advanced detector injection systems, an in-vacuum remotely controlled mode matching telescope composed of moving lenses and mirrors allows the compensation of the Radius of Curvature (RoC) of the laser beam as well as any tilt. Thus the whole dynamic range of the TDM can be dedicated to higher order mode corrections. Before starting to use the system, we take a reference wavefront when no current is applied on the actuators. Then, all the measurements are made with respect to this reference. When using the TDM the components of RoC and tilt are subtracted numerically from the measured wavefront. The RoC that was typically subtracted is of the order of $100 \mathrm{~m}$ to $50 \mathrm{~m}$ over the $7 \mathrm{~mm}$ pupil. The tilt subtracted was about $5 \mu \mathrm{rad}$.

\section{Actuator characterization}

Each actuator driven with a constant current produces a phase deformation that is its individual spatial response and may therefore be considered as the actuator's influence function. In figure 4 we show examples of these responses for three different actuators. The differences in amplitude between actuators is mainly due to the large difference in resistor values.

In figure 5 we compare the profile of the central actuator influence function with that simulated by finite element analysis. We see that the amplitude of the response is roughly equivalent for a simulated absorbed power of $9.4 \mathrm{~mW}$. The total calculated dissipated power of the actuator is $27 \mathrm{~mW}$. We may therefore conclude from this comparison that approximately $35 \%$ of the total power dissipated by the resistor is absorbed by the substrate, the remaining $65 \%$ is presumably dissipated by convection on the backside of the heater array. We also see that the experimental profile is wider than that of the simulation. This could be attributed to conduction across the surface of the heater array which is not taken into account in the simulation. The amplitude of deformation of the TDM at the maximum of the actuation command may be considered as the stroke of the actuator. In the case of figure 5 the stroke is of $41 \mathrm{~nm} \mathrm{PtV}$.

A fundamental requirement for a deformable mirror is that the actuator response to a command be linear. The Root Mean Square (RMS) of the phase deformation is measured 
while increasing the current injected into the central actuator. In figure 6 we see the resulting RMS as a function of dissipated power calculated from the current. We see that for low powers we are limited by the precision of the wavefront sensor, but for higher powers we can state that the response of the actuator is proportional to the square of the injected current.

The temporal behavior of the actuator may be characterized by measuring the evolution of the phase deformation after switching on the current to a few $\mathrm{mA}$. The experimental measurements show that the response can be modeled as a second order system. It has been observed that the time constants depend greatly on the thermal contact between the heater array and the mirror substrate. The use of thermal paste yields time constants of $\tau_{1}=2$ $\mathrm{s}$ and $\tau_{2}=0.2 \mathrm{~s}$ which is about three times faster than when using glue as the thermal contact. The measured time constants indicate that the actuation system should be driven at frequencies lower than $0.15 \mathrm{~Hz}$. As the response of the actuator is proportional to the actuator power, the final influence functions are normalized by the square of the injected current.

\section{Control algorithm of the TDM}

\section{A. Error minimization with a least-square algorithm}

Since the device is linear, the action of the TDM can be described by the matrix $M$ which contains all the influence functions. To correct an aberrated wavefront it is necessary to produce the opposite phase deformation with the active reflecting device. The control of the device is therefore reduced to find the best set of commands to reproduce a desired target wavefront $\psi_{t}$. The goal is the minimization of the difference $\psi_{e}$ between the TDM action and the desired wavefront:

$$
\psi_{e}=\psi_{t}-M a
$$

where $a$ is the vector of the correction value for each of the resistors. The standard solution to this problem, often used in adaptive optics applications, is to find the least square error solution, i.e. the one that minimize the error:

$$
\epsilon=\left\|\psi_{t}-M a\right\|_{2}^{2}
$$

This approach yields a non bounded vector that can have positive or negative values. However the TDM is a one-directional actuation system, since we can only heat the substrate. This

issue is normally addressed operating with a constant added to each command. The so called bias operation consists of putting the actuators at the middle of their dynamic range and to make them fit a simple shape (piston or focus) before the calibration of the system. But both 
these methods are not taking into account the physical actuation limits of the device: the least square solution might still give solutions out of the actuation range. These commands will be clipped resulting in inaccuracies or even instabilities or losses of control in the system when the actuators start to saturate.

In order to improve the control of our system and to efficiently exploit the entire dynamic range, we decided to use an algorithm [30] that allows us to choose lower bounds, $l$ and upper bounds, $u$ for the command values $a$ :

$$
l_{i} \leq a_{i} \leq u_{i} \quad \text { with } i=1 \ldots 61
$$

where $i$ indexes all the resistors. The optimal solution is still considered the one that minimizes the error of Eq. (2). Its gradient must be zero at the optimal point, except for those $a_{i}$ that are at the boundary: for those the corresponding gradient component must be such that the decreasing direction points beyond the limit, or in other words to improve the error it would be necessary to relax one of the constraints of Eq. (3).

These kinds of search algorithms are typically much more computationally demanding than the simple least-square solution, taking about one third of a second for a standard PC. This is not an issue in our application, since the time available for the computation is long enough.

\section{B. Closed-loop control}

The constrained minimization algorithm can be used to find the best estimate of the correction commands needed to reproduce a target wavefront. If all the influence functions were perfectly known, one single computation would be enough to find the optimal correction. To make the system more robust against uncertainties in the measured influence function or changes in the system response, we implemented an iterative, closed-loop scheme. At the beginning the best correction is computed as previously explained and sent to the TDM. After the settling time the residual wavefront $\psi_{r}$ is measured. This is used as the new target for the algorithm. Since the new correction must be added to the previous one, the boundaries are properly shifted in order to ensure that the total correction remains inside the allowed region. This iteration can be repeated as many times as needed. As will be discussed in subsequent sections, it allowed an improvement in the actuation efficiency and the capability of tracking time-changing conditions.

\section{Wavefront correction characterization}

\section{A. Efficiency and accuracy of the correction}

The Zernike polynomials form an orthogonal basis over the unit circular aperture. They are often used to describe the low-spatial frequency wavefront distortions as the first polynomials correspond to the principal aberrations occurring in optical systems [29]. 
In order to reduce the numerical complexity of the algorithm that computes the correction, the influence matrix, obtained from the calibration of the 61 actuators, and the measured wavefronts are decomposed into Zernike modes:

$$
\psi=\sum_{i=1}^{N} \alpha_{i} Z_{i}
$$

where $\alpha_{i}$ is the coefficient of the Zernike mode $i . N$ is the number of Zernike modes used in the decomposition. The basis is normalized such that the RMS of any wavefront is given by:

$$
R M S_{\psi}=\sqrt{\sum \alpha_{i}^{2}}
$$

Therefore in our performance analysis each target Zernike mode $i$ is described by the corresponding coefficient $\alpha_{t_{i}}$. In a real system application the target wavefront corresponds to the beam aberration to be corrected. For the characterization of the TDM we decided to use target wavefronts composed of single Zernike modes. In this way, as it will be shown later, we are able to derive quantitative estimates of the TDM performance. Since the generation of higher Zernike modes is limited by the spatial frequency of the actuator pattern, we restricted our study to the first 20 modes (Zernike polynomials with order smaller than 5). As we mentioned previously in section 2.C, we will neglect the correction of tilt and focus which can be achieved using simple optical elements.

Each Zernike mode generated by the mirror is characterized by its spatial amplitude distribution as well as the maximum deformation (Peak to Valley (PtV)) and the RMS values of this distribution. The difference between the Zernike polynomial to reproduce and the wavefront generated by the TDM actuation is called the residual phase image. It corresponds to the wavefront $\psi_{e}$ to minimize. The results shown are obtained in a closed-loop control configuration. In figure 7 we show an example of the evolution of residual RMS as a function of the number of iterations. Each iteration allows a settling time of $10 \mathrm{~s}$. After less than 10 iterations the resulting phase image is stable and corresponds to the best solution.

Figure 8(b) shows an example of the Zernike mode $Z_{3}^{-3}$ generated in this way. In figure 8 (c) we see the residual wavefront after TDM correction. This residual phase shape has a dominant spatial frequency of $0.5 \mathrm{~mm}^{-1}$ which is directly related to the spatial frequency of the actuator array. This effect is generally referred to as the actuator footprint.

The quality of the Zernike mode generated by the TDM can be evaluated according to two criteria. The efficiency $E$ determines if the amplitude of the reproduced mode is close to the amplitude of the target mode. To evaluate this first criterion, we compare the coefficient $\alpha_{7}$ of the generated Zernike $Z_{3}^{-3}$ (that is the $7^{\text {th }}$ polynomial of the Zernike basis) to the 
coefficient of the target mode $\alpha_{t_{7}}$ :

$$
E=1-\frac{\left|\alpha_{t_{7}}-\alpha_{7}\right|}{\alpha_{t_{7}}}
$$

The closer to 1 this parameter, the greater the efficiency of the TDM to reproduce the target mode. For low order modes, the efficiency will be principally limited by the actuator saturation. In this example the efficiency is $99.8 \%$. This high efficiency indicates that we are working within the dynamic range of the TDM.

The second criterion concerns the generation of unwanted Zernike modes by the TDM. We define the accuracy $A$ of the mode generation as:

$$
A=\frac{\alpha_{7}}{\sqrt{\sum_{i=1}^{20} \alpha_{i}^{2}}}
$$

The closer to 1 this parameter, the fewer unwanted modes generated by the TDM. We obtain 99,1\% of accuracy for the Zernike mode $Z_{3}^{-3}$ at $50 \mathrm{~nm}$ PtV. This indicates that the mode coupling is relatively low: the Zernike mode $Z_{3}^{-3}$ can be generated independently of others. The experimental results obtained in closed-loop control are shown in figure 9 for Zernike modes up to 20 for Zernike target modes having a $10 \mathrm{~nm}$ RMS.

The accuracy is very high for all the polynomials tested. The minimum value obtained is for the mode 18. We may therefore conclude that the spatial resolution of the TDM is sufficient to correct up to the $20^{\text {th }}$ Zernike mode with minimal mode coupling. For modes below to the third order (modes 3 to 9), the efficiency is also close to 1 with a minimum of 98.5 at which point the actuation is limited by the actuator footprint. The efficiency is globally decreasing for the 4 th and 5 th order (modes 13 to 20). It reaches a minimum for the mode 18 at $46.6 \%$. The reduced efficiency is attributed to the increasing number of saturated actuators, thereby confirming that for higher order Zernikes the TDM is limited by its dynamic range.

We checked experimentally that the wavefront response to the TDM correction is linear: the effect of switching on two actuators is equal to the superposition of the effect of the single actuators. This is clearly only true when the actuators are not saturated.

\section{B. Mismatch correction}

These experimental results show that the TDM is able to generate Zernike polynomials up to the mode $20^{\text {th }}$. The limiting factors are the power of actuation and the residual footprint: the power of actuation restricts the linear response range of the TDM while the residual footprint limits the precision of the mode reproduction. In terms of mode matching applications, the 
target wavefront corresponds to the initial mismatch to be corrected; the residual phase corresponds to the aberrations that will limit the best achievable matching. We want to determine how the correction produced by the TDM can improve the matching.

The generation of the modes of the 2 nd and 3rd order demonstrate a linear working range of almost $20 \mathrm{~nm}$ RMS (see figure 10 (a) ). Using an overlap integral calculation, we can see that they are equivalent to a mismatching of around $3 \%$ for each mode. The RMS value of the residual phase images from the mode generation are given in figure 10 (b). The RMS value of the residual image is between $1.2 \mathrm{~nm}$ and $4 \mathrm{~nm}$ RMS for the Zernike orders 2 and 3. From these results, we can again deduce by an overlap integral calculation that if we have a mismatching due to aberrations composed of the second or third order Zernike polynomials inside the tested range, the TDM is able to correct them to reach a matching better than 99\% (see table 11). It means that in the limits of its dynamic range the TDM can correct these aberrations within the specifications for Advanced Virgo.

\section{Discussion and Conclusion}

We demonstrated that the thermally deformable mirror presented here can generate efficiently Zernike polynomials up to the $5^{\text {th }}$ order, opening the way for high resolution aberration compensation using this new technology. At present time, the practical use of the device is limited by its dynamic range that limits corrections to about 30nm RMS for Zernikes up to the 3rd order. For Zernikes up to the 3rd order, the TDM can reduce the amplitude of RMS aberrations by up to a factor 5. Taking the example of Advanced Virgo, the thermal distortions are expected to create a mismatching of $10 \%$ that would correspond to a needed correction of about $50 \mathrm{~nm}$ RMS dominated by low order aberrations. Extending the dynamic range of the TDM by a factor 2 would allow to reduce the mismatching to around $1 \%$ and therefore fulfill Advanced Virgo requirements. It would even enable to improve further the thermal distortions by correcting higher order aberration and therefore reach a matching of better than $99 \%$. The use of a TDM for mode matching correction inside advanced GW interferometers will require not only controlling the phase of the laser beam but also its intensity distribution. This could be done by using two different TDMs placed on the beam path at different Gouy phases. This new technology is therefore very promising for advanced GW interferometers where it could be used not only for coupling the input beam into the interferometer but also for coupling the dark fringe beam into the output filtering cavities. Furthermore, the use of the TDM could be even more important for future GW detectors that will use frequency dependent squeezing techniques. Indeed, the efficiency of this method will be critically dependent on mode matching quality on the filter cavities [31]. Finally, as the TDM could be used with various type of substrates, it could be easily integrated onto many other laser experiments, where the aberrations to be corrected are slowly varying and 
where the noise requirements are stringent.

\begin{abstract}
Aknowledgments
We would like to warmly thank the Stevenage Circuits company (Stevenage Circuits Ltd,Caxton Way, Stevenage, SG1 2DF, UK,

http://www.stevenagecircuits.co.uk) for their cordial work and assistance in the construction of the resistor array for this first prototype.
\end{abstract}




\section{References}

1. P. Hello and J.Y. Vinet, "Analytical models of thermal aberrations in massive mirrors heated by high power laser beams", J. Phys. France 51 (12) 1267-1282 (1990)

2. P. Hello and J.Y. Vinet, "Analytical models of transient thermoelastic deformations of mirrors heated by high power cw laser beams", J. Phys. France 51 (12) 2243-2261 (1990)

3. W. Winkler, K. Danzmann ,A. Rudiger and R. Schilling, Phys. "Heating by optical absorption and the performance of interferometric gravitational-wave detectors", Phys. Rev. A 447022 (1991)

4. J.-Y. Vinet, "On Special Optical Modes and Thermal Issues in Advanced Gravitational Wave Interferometric Detectors", Living Rev. Relativity 12 (2009)

5. H. Luck, K.-O. Muller, P. Aufmuth and K. Danzmann, "Correction of wavefront distortions by means of thermally adaptive optics", Optics Communications 175, 275-287 (2000)

6. P. Hello, "Compensation for thermal effects in mirrors of gravitational wave interferometers", Eur. Phys. J. D 15, 373-383 (2001)

7. R. Lawrence, M. Zucker, P. Fritschel, P. Marfuta, D. Shoemaker "Adaptive thermal compensation of test masses in advanced LIGO", Class. Quantum Grav. 19 1803-1812 (2002)

8. J. Degallaix, C. Zhao, L. Ju and D. Blair, "Thermal lensing compensation for AIGO high optical power test facility", Class. Quantum Grav. 21, S903 (2004)

9. H. Luck, A. Freise, S. Gobler, S. Hild, K. Kawabe and K. Danzmann, "Thermal correction of the radii of curvature of mirrors for GEO 600", Class. Quantum Grav. 21 S985 (2004)

10. R. Lawrence, D. Ottaway, M. Zucker, P. Fritschel, "Active correction of thermal lensing through external radiative thermal actuation", Opt. Lett. 29(22), 2635-2637 (2004)

11. V. Quetschke, J. Gleason, M. Rakhmanov, J. Lee, L. Zhang, K. Yoshiki Franzen, C. Leidel, G. Mueller, R. Amin, D. B. Tanner, and D. H. Reitze, "Adaptive control of laser modal properties", Opt. Lett. 31,217-219 (2006)

12. Muzammil A. Arain, Volker Quetschke, Joseph Gleason, Luke F. Williams, Malik Rakhmanov, Jinho Lee, Rachel J. Cruz, Guido Mueller, D. B. Tanner, and David. H. Reitze, "Adaptive beam shaping by controlled thermal lensing in optical elements", Appl. Opt. 46, 2153-2165 (2007)

13. R. Day et al., "Central Heating Radius of Curvature Correction for use in large scale Gravitational Wave Interferometers", submitted to Class. Quantum Grav. (2012)

14. The Virgo Collaboration, "Advanced Virgo Technical design report", Virgo collaboration internal document (2012)

15. The LIGO Scientific Collaboration, "A gravitational wave observatory operating beyond 
the quantum shot-noise limit", Nature Physics 7, 962-965 (2011)

16. M. Abernathy et al., "Einstein gravitational wave Telescope conceptual design study", ET-0106C-10, https://tds.ego-gw.it/ql/?c=7954 (2011)

17. G. Mueller, Q. Shu, R. Adhikari, D. B. Tanner, D. Reitze, D. Sigg, N. Mavalvala and J. Camp, "Determination and optimization of mode matching into optical cavities by heterodyne detection", Opt. Lett. 25. 266-268 (2000)

18. G. Mueller, R. S. Amin, D. Guagliardo, D. McFeron, R. Lundock, D. H. Reitze and D. B. Tanner, "Method for compensation of thermally induced modal distortions in the input optical components of gravitational wave interferometers", Class. Quantum Grav. 19 1793-1801 (2002)

19. E. Calloni, J. T. Baker, F. Barone, R. DeRosa, L. Di Fiore, L. Milano, and S. R. Restaino, "Adaptive optics approach for prefiltering of geometrical fluctuations of the input laser beam of an interferometric gravitational waves detector", Rev. Sci. Instrum. 74, 2570 (2003)

20. S. Avino, "Adaptive Optics Techniques for Gravitational Wave Interferometers", PhD thesis, Universita degli studi di Napoli Federico II (2006)

21. M. Arain, W. Korth, L. Williams, R. Martin, G. Mueller, D. Tanner, and D. Reitze, "Adaptive control of modal properties of optical beams using photothermal effects," Opt. Express 18, 2767-2781 (2010).

22. B. Canuel, R. Day, E. Genin, P. La Penna and J. Marque, "Wavefront aberration compensation with thermally deformable mirror", Class. Quantum Grav. 29, 085012 (2012)

23. S. Velghe , J. Primot and N. Guerineau, M. Cohen and B. Wattellier ,"Wave-front reconstruction from multidirectionalphase derivatives generated by multilateral shearing interferometers", Optics Letters 30 (3), 245-247 (2005)

24. E. Fernandez, L. Vabre, B. Hermann, A. Unterhuber, B. Povazay, and W. Drexler, "Adaptive optics with a magnetic deformable mirror: applications in the human eye," Opt. Express 14, 8900-8917 (2006)

25. R. Heimsten, D. MacMynowski, T. Andersen, and M. Owner-Petersen, "Concept, modeling, and performance prediction of a low-cost, large deformable mirror," Appl. Opt. 51, 515-524 (2012)

26. J. Sung, T.M. Jeong, S. K. Lee, T. J. Yu, I. W. Choi, J. Lee, "Analysis of Thermal Aberrations in the Power Amplifiers of a 10-Hz 100-TW Ti:sapphire Laser", Journal of the Korean Physical Society, 55, 495-500 (2009)

27. J. Alda, G. D. Boreman, "Zernike-based matrix model of deformable mirrors: optimization of the aperture size", Appl. Opt. 32, 2431-2438 (1993)

28. S. Verpoort, U. Wittrock, "Actuator patterns for unimorph and bimorph deformable mirrors", Appl. Opt. 49, G37-G46 (2010) 
29. M. Born, E. Wolf, Principle of optics (Cambridge University Press, 1999)

30. T. F. Coleman and Y. Li, "A Reflective Newton Method for Minimizing a Quadratic Function Subject to Bounds on Some of the Variables", SIAM Journal on Optimization, Vol. 6, Number 4, pp. 1040-1058 (1996)

31. T. Corbitt, N. Mavalvala, and S. Whitcomb,"Optical cavities as amplitude filters for squeezed fields", Phys. Rev. D 70, 022002 (2004) 


\section{List of Figure Captions}

Fig. 1. Schematic representation of the TDM principle: the wavefront of a laser beam is modified by reflection on the back side of a substrate for which its temperature is tuned by an array of resistors.

Fig. 2. (a) Scheme of the prototype array with the 61 resistors in blue and the pupil of observation defined by the area enclosed by the red circle. The resistors are separated by a dead space of $100 \mu \mathrm{m}$. (b) Photograph of the prototype array

Fig. 3. Schematic of the adaptive optical setup of TDM characterization. The continuous wave laser beam $(\mathrm{CW})$ is directly sent to the TDM and the phase deformations occurring in the pupil area are analyzed by a wavefront sensor.

Fig. 4. Three experimental actuator responses of the TDM (colorscale in $\mathrm{nm}$ ) at $5 \mathrm{~mA}$ with the resistor values and their corresponding position in the prototype array in red.

Fig. 5. Experimental phase deformation created by the central actuator dissipating a power of $27 \mathrm{~mW}$ (blue) compared with results from finite element analysis simulation for an absorbed power of $9.4 \mathrm{~mW}$ (red). The simulation is fitted to have the same area under the curve than the experimental data (same total power).

Fig. 6. RMS of the phase deformation as a function of power dissipated by the central resistor in the substrate. The dots are the experimental measurement values. The blue line corresponds to the best linear fit of the experimental data. The slope is indicated by the red line and the offset by the green line: it corresponds to the noise level of the wavefront sensor, that is lowered by average of experimental data.

Fig. 7. RMS of the residual image in a closed-loop control for the target mode $Z_{3}^{-3}$ at 50 $\mathrm{nm} \mathrm{PtV}$. The first iteration corresponds to the residual RMS that may be obtained in an open-loop control.

Fig. 8. Zernike mode $Z_{3}^{-3}$ generated by the TDM over a pupil of $7 \mathrm{~mm}$ (b). A RoC of 350 $\mathrm{m}$ and a horizontal tilt of 5 microrad have been numerically subtracted. The difference with the target Zernike mode (a) is the residual phase image (c) (colorscales are in $\mathrm{nm}$ ).

Fig. 9. Characterization of the modes generated by the TDM: for each mode the efficiency is given in the green bars and the accuracy is given in the blue bars. All the Zernike target modes have an RMS of $10 \mathrm{~nm}$.

Fig. 10. Full scan of the Zernike modes from the 2nd and 3rd order: (a) RMS value of the Zernike produced by the mirror, (b) RMS value of the residual image. 


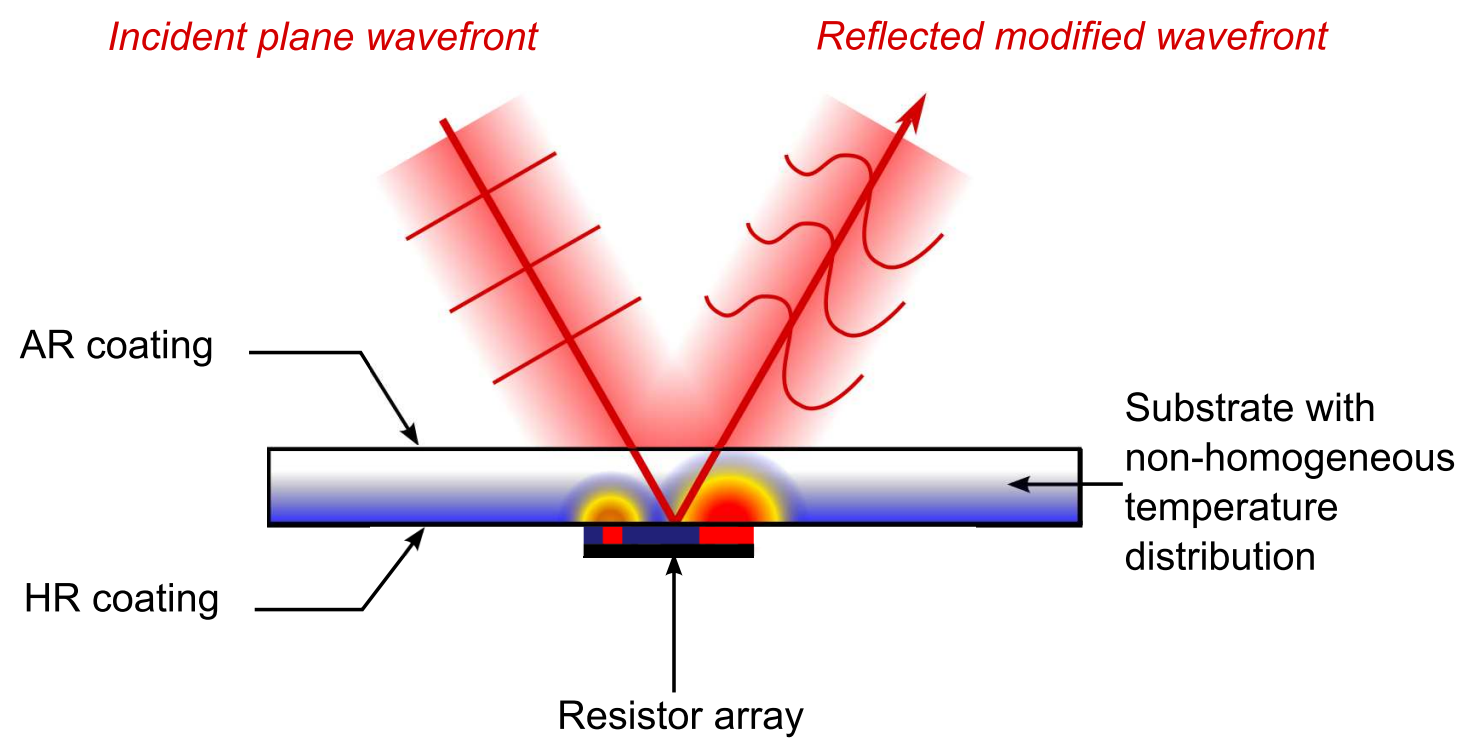

Figure 1. Schematic representation of the TDM principle: the wavefront of a laser beam is modified by reflection on the back side of a substrate for which its temperature is tuned by an array of resistors. kasprzack_fig1 


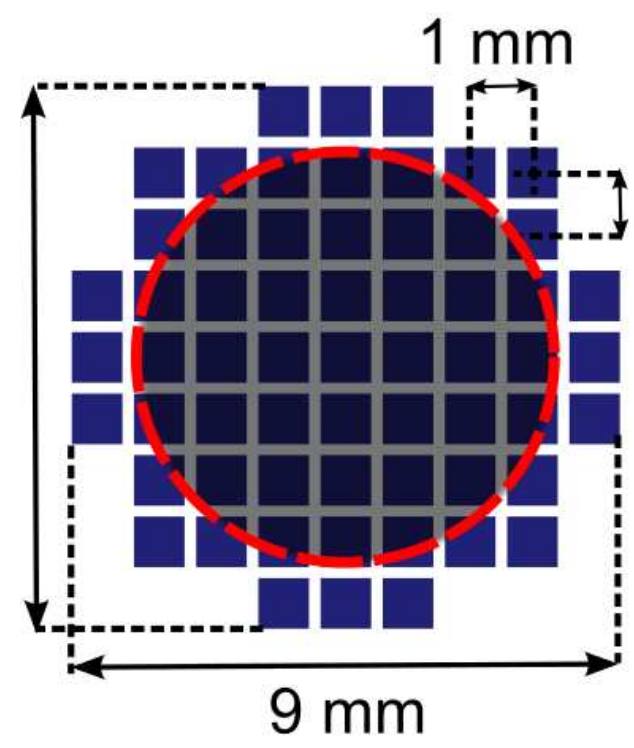

(a)

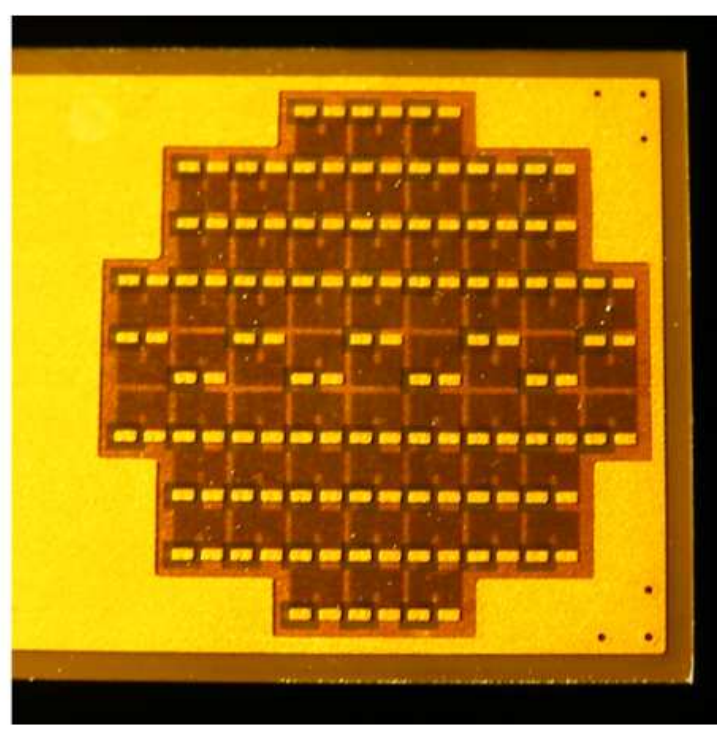

(b)

Figure 2. (a) Scheme of the prototype array with the 61 resistors in blue and the pupil of observation defined by the area enclosed by the red circle. The resistors are separated by a dead space of $100 \mu \mathrm{m}$. (b) Photograph of the prototype array. kasprzack_fig2 


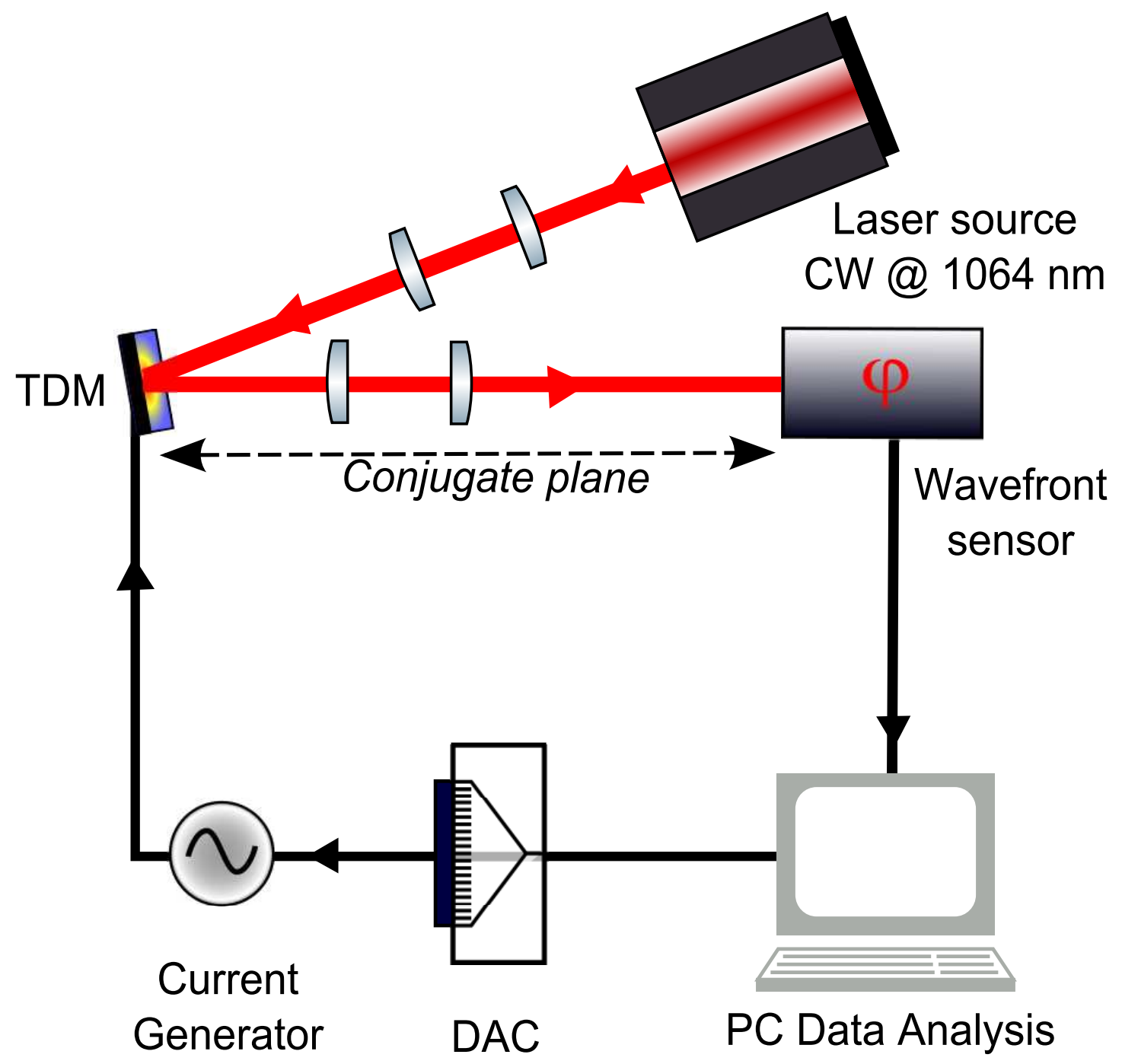

Figure 3. Schematic of the adaptive optical setup of TDM characterization. The continuous wave laser beam (CW) is directly sent to the TDM and the phase deformations occurring in the pupil area are analyzed by a wavefront sensor.kasprzack_fig3 

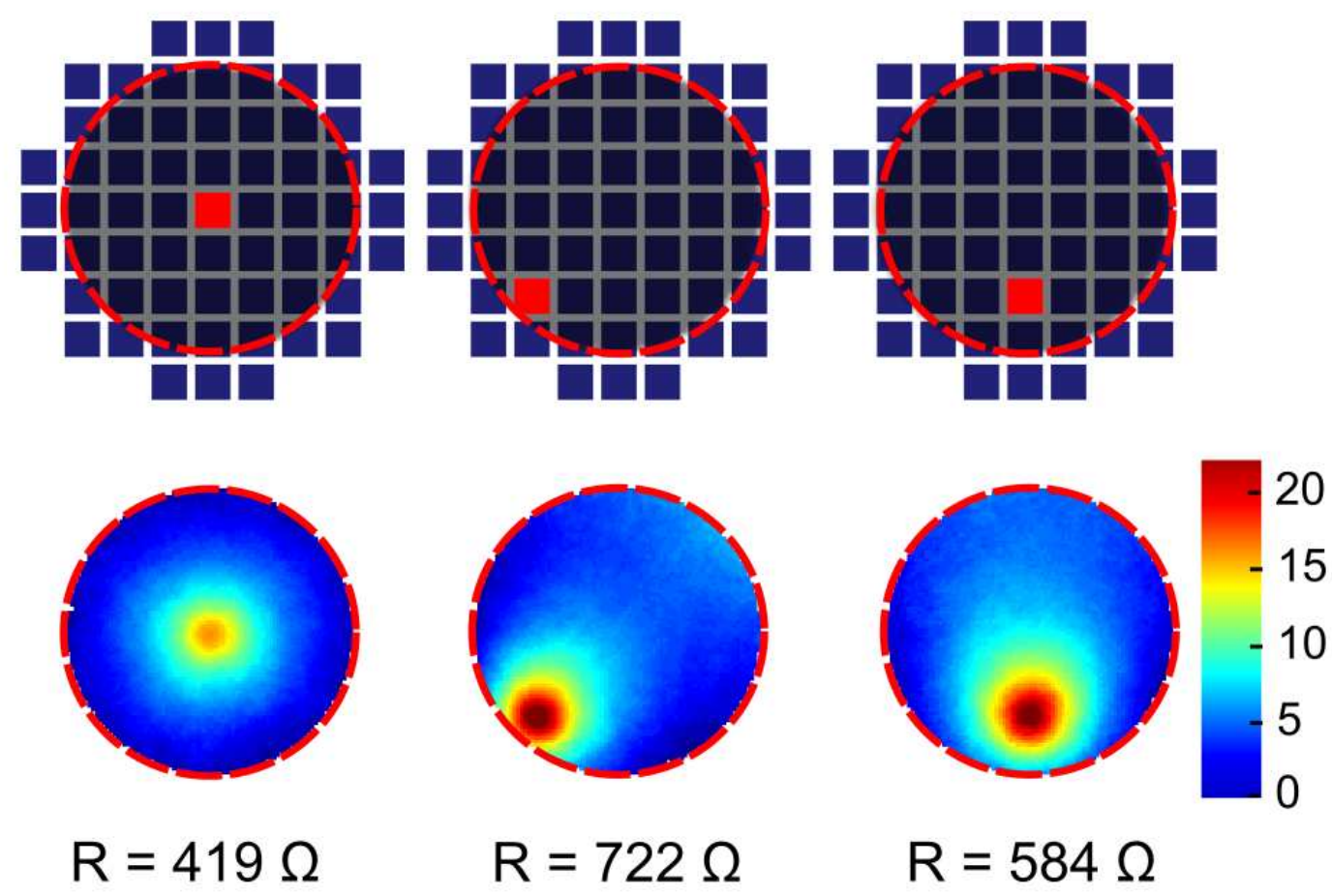

$\mathrm{R}=584 \Omega$

Figure 4. Three experimental actuator responses of the TDM (colorscale in $\mathrm{nm}$ ) at $5 \mathrm{~mA}$ with the resistor values and their corresponding position in the prototype array in red. kasprzack_fig4 


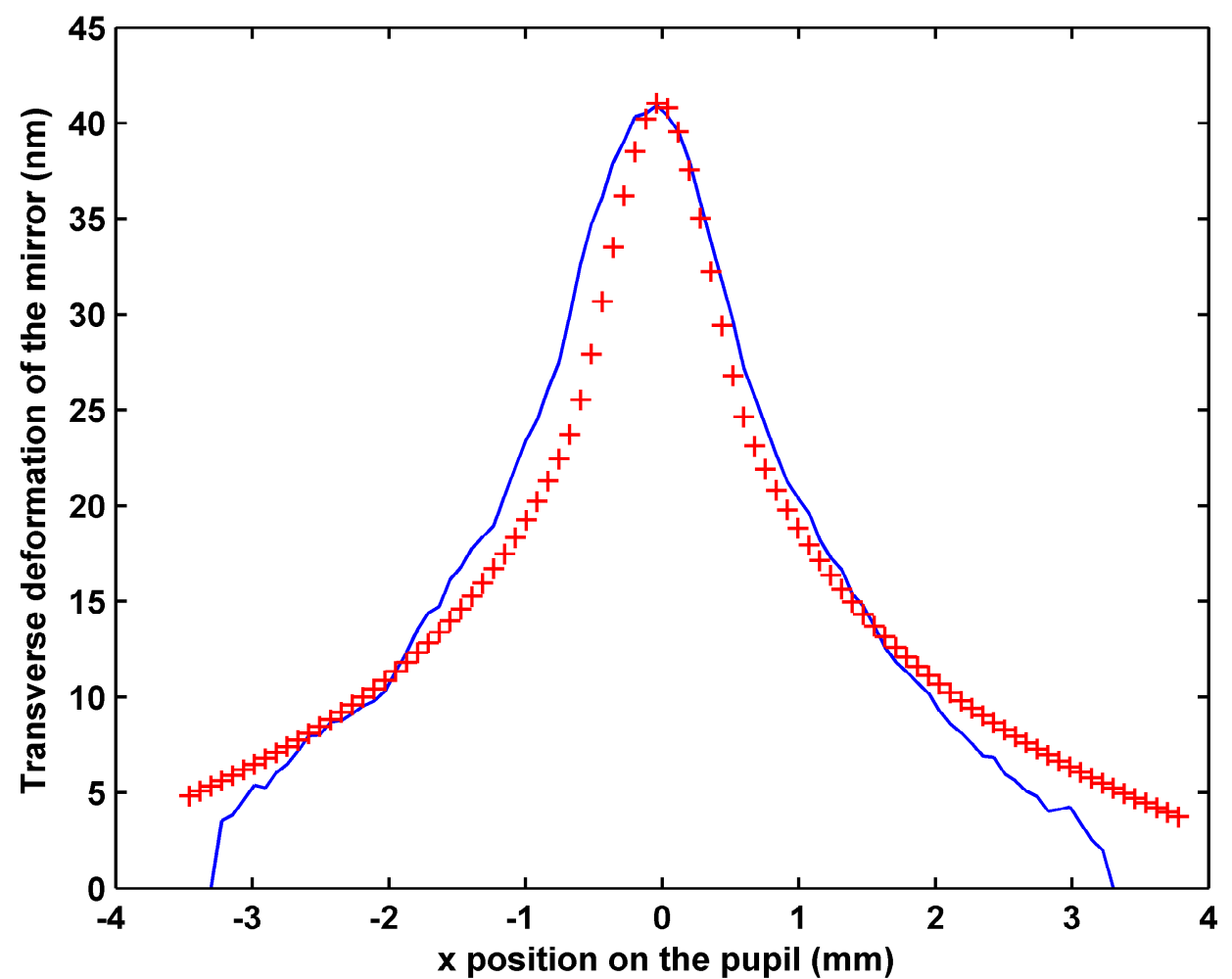

Figure 5. Experimental phase deformation created by the central actuator dissipating a power of $27 \mathrm{~mW}$ (blue) compared with results from finite element analysis simulation for an absorbed power of $9.4 \mathrm{~mW}$ (red). The simulation is fitted to have the same area under the curve than the experimental data (same total power). kasprzack fig5 


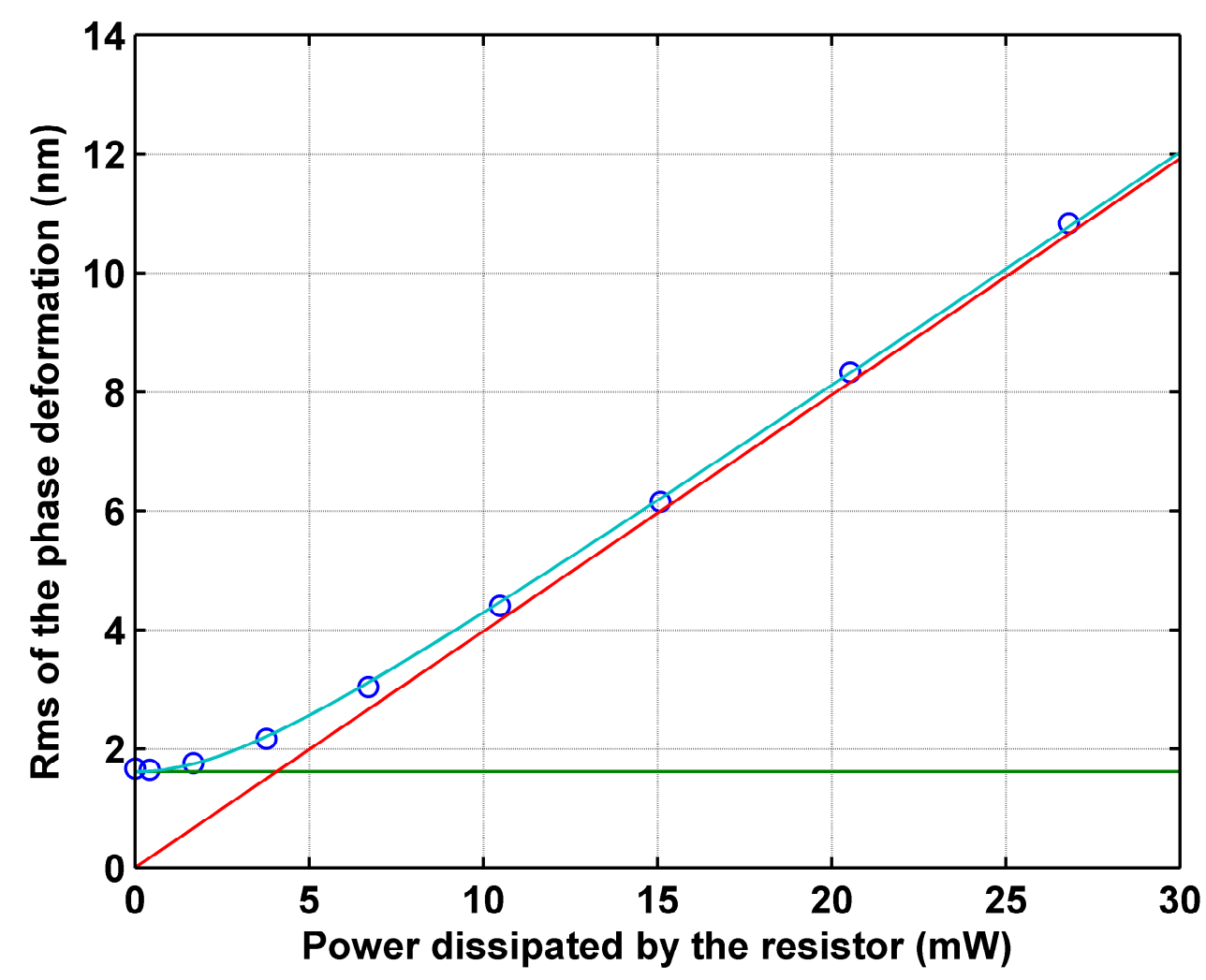

Figure 6. RMS of the phase deformation as a function of power dissipated by the central resistor in the substrate. The dots are the experimental measurement values. The blue line corresponds to the best linear fit of the experimental data. The slope is indicated by the red line and the offset by the green line: it corresponds to the noise level of the wavefront sensor, that is lowered by average of experimental data. kasprzack fig6 


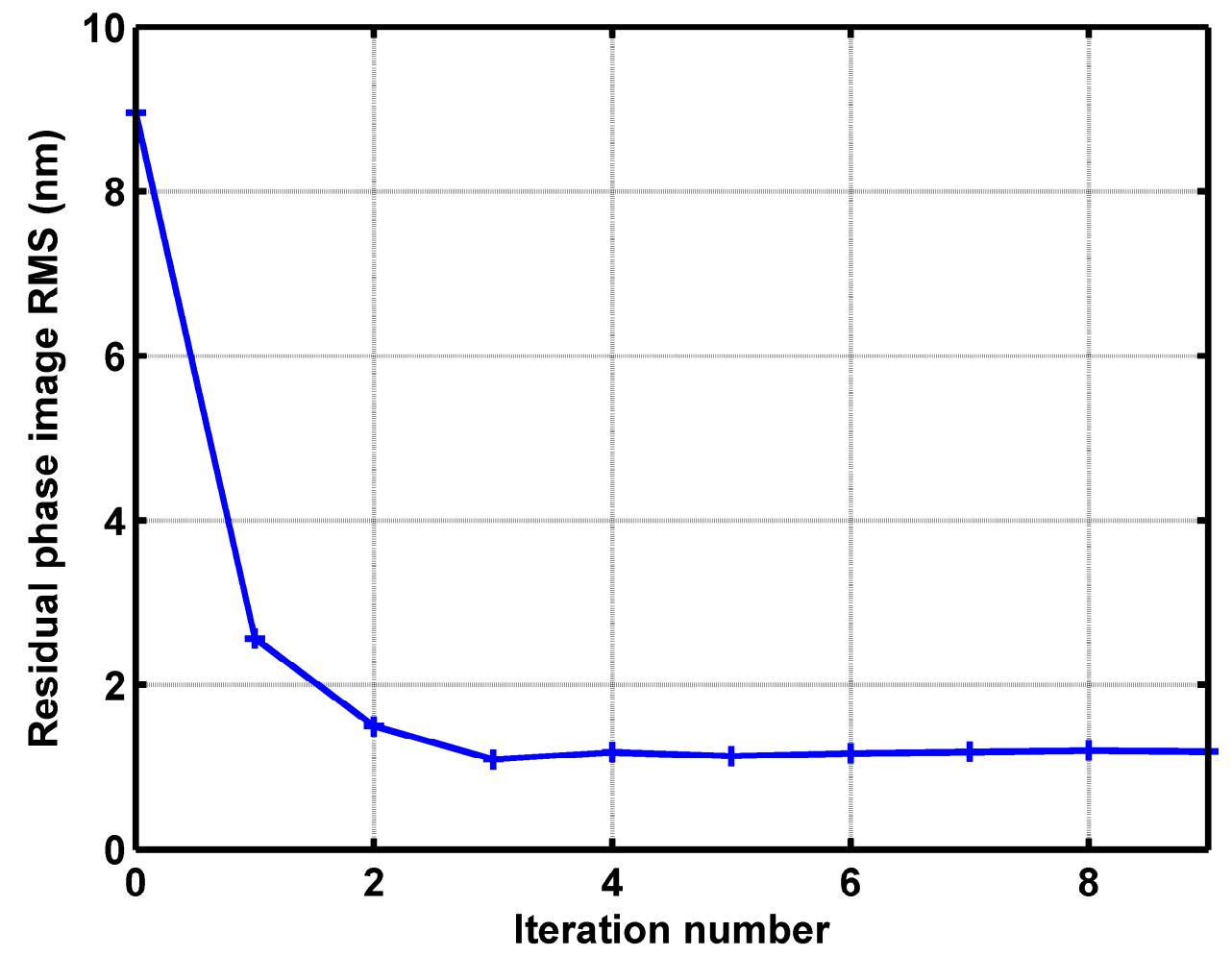

Figure 7. RMS of the residual image in a closed-loop control for the target mode $Z_{3}^{-3}$ at $50 \mathrm{~nm} \mathrm{PtV}$. The first iteration corresponds to the residual RMS that may be obtained in an open-loop control. kasprzack_fig7 

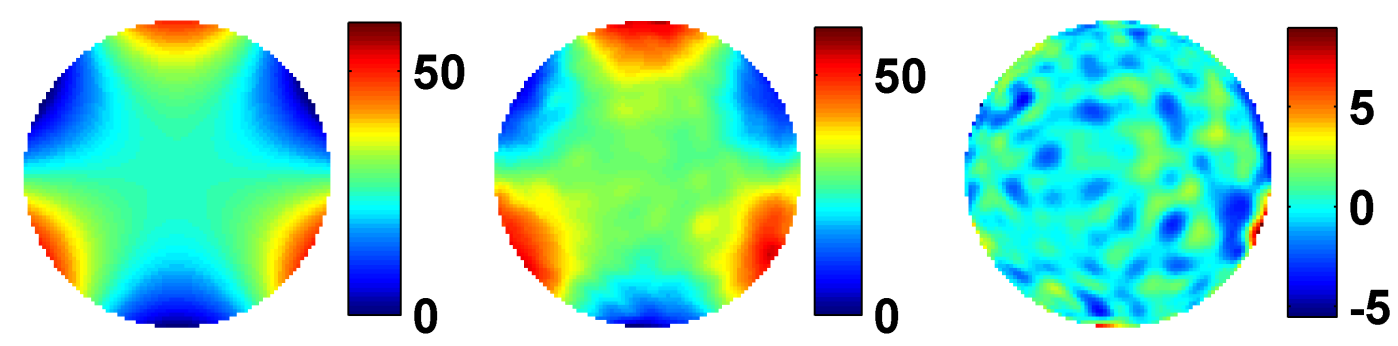

Zernike mode target Zernike mode mirror

$\mathrm{PtV}=50.0 \mathrm{~nm}$

$\mathrm{PtV}=55.7 \mathrm{~nm}$

Residual shape

$\mathrm{RMS}=9.0 \mathrm{~nm}$

(a)

$\mathrm{RMS}=9.0 \mathrm{~nm}$

(b)
$\mathrm{PtV}=14.5 \mathrm{~nm}$

$\mathrm{RMS}=1.2 \mathrm{~nm}$

(c)

Figure 8. Zernike mode $Z_{3}^{-3}$ generated by the TDM over a pupil of $7 \mathrm{~mm}$ (b). A RoC of $350 \mathrm{~m}$ and a horizontal tilt of 5 microrad have been numerically subtracted. The difference with the target Zernike mode (a) is the residual phase image (c) (colorscales are in $\mathrm{nm}$ ). kasprzack_fig8 


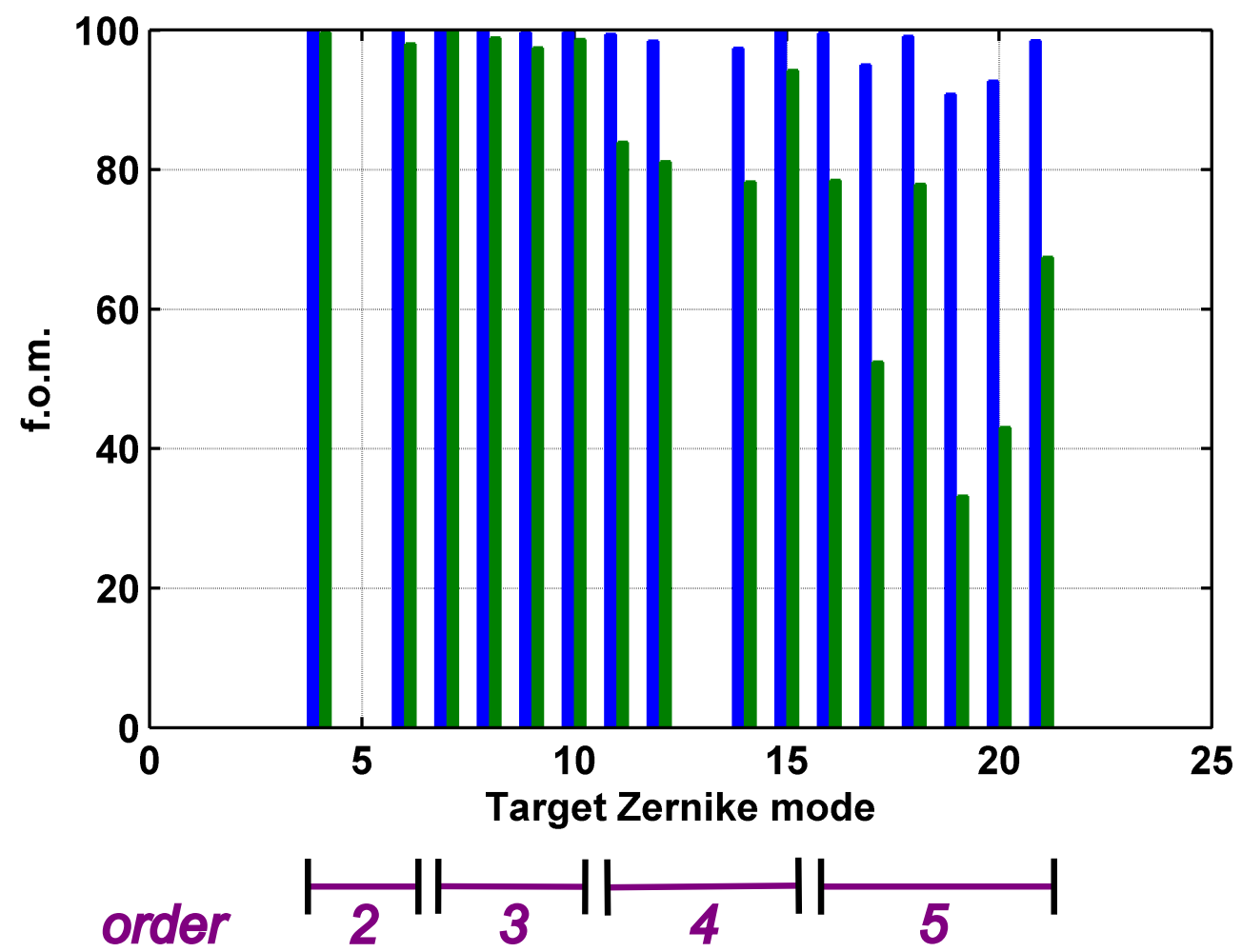

Figure 9. Characterization of the modes generated by the TDM: for each mode the efficiency is given in the green bars and the accuracy is given in the blue bars. All the Zernike target modes have an RMS of $10 \mathrm{~nm}$. kasprzack_fig9 


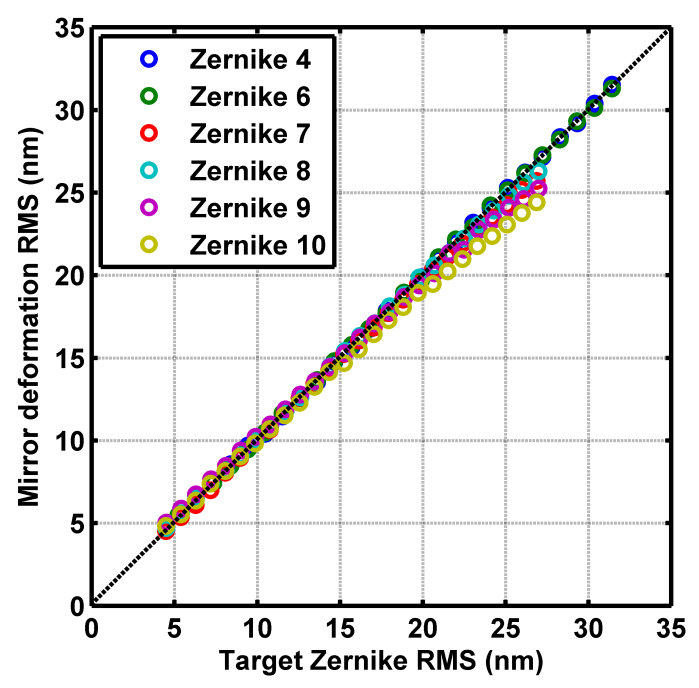

(a)

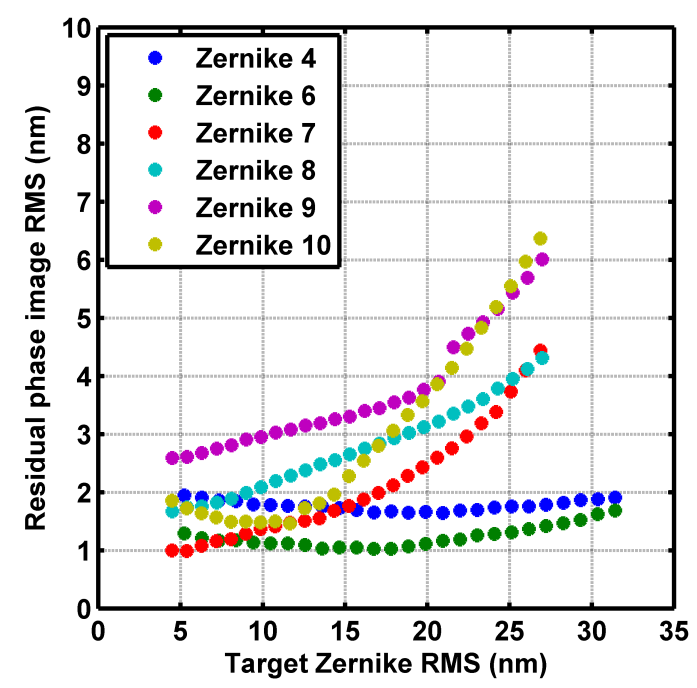

(b)

Figure 10. Full scan of the Zernike modes from the 2nd and 3rd order: (a) RMS value of the Zernike produced by the mirror, (b) RMS value of the residual image. kasprzack_fig10 


\begin{tabular}{|c|c|c|c|c|c|c|}
\hline \multicolumn{7}{|c|}{ - Extrapolated results of the TDM correction - } \\
\hline Zernike mode & 4 & 6 & 7 & 8 & 9 & 10 \\
\hline Initial matching (\%) & 0.9610 & 0.9610 & 0.9708 & 0.9721 & 0.9721 & 0.9708 \\
\hline Matching after correction (\%) & 0.9999 & 0.9999 & 0.9992 & 0.9993 & 0.9986 & 0.9983 \\
\hline
\end{tabular}

Figure 11. Table of the correction of the mode matching by extrapolation of the experimental results. 